\author{
Marquette University \\ e-Publications@Marquette
}

Biomedical Engineering Faculty Research and

Publications

Biomedical Engineering, Department of

3-15-2002

\title{
Histologic Evaluation of the Efficacy of rhBMP-2 Compared With Autograft Bone in Sheep Spinal Anterior Interbody Fusion
}

\author{
Harvinder Sandhu \\ Cornell University Medical College \\ Jeffrey M. Toth \\ Marquette University, jeffrey.toth@marquette.edu \\ Ashish Diwan \\ Cornell University Medical College \\ H. B. Seim III \\ Colorado State University, Fort Collins \\ Linda E.A. Kanim \\ UCLA School of Medicine
}

See next page for additional authors

Follow this and additional works at: https://epublications.marquette.edu/bioengin_fac

Part of the Biomedical Engineering and Bioengineering Commons

\section{Recommended Citation}

Sandhu, Harvinder; Toth, Jeffrey M.; Diwan, Ashish; Seim, H. B. III; Kanim, Linda E.A.; Kabo, J. Michael; and Turner, A. Simon, "Histologic Evaluation of the Efficacy of rhBMP-2 Compared With Autograft Bone in Sheep Spinal Anterior Interbody Fusion" (2002). Biomedical Engineering Faculty Research and Publications. 560.

https://epublications.marquette.edu/bioengin_fac/560 
Authors

Harvinder Sandhu, Jeffrey M. Toth, Ashish Diwan, H. B. Seim III, Linda E.A. Kanim, J. Michael Kabo, and A. Simon Turner

This article is available at e-Publications@Marquette: https://epublications.marquette.edu/bioengin_fac/560 
Marquette University

e-Publications@Marquette

\section{Biomedical Engineering Faculty Research and Publications/College of Engineering}

This paper is NOT THE PUBLISHED VERSION; but the author's final, peer-reviewed manuscript. The published version may be accessed by following the link in the citation below.

Spine, Vol. 27, No. 6 (March, 2002): 567-575. Permalink. This article is @ Lippincott Williams \& Wilkins, Inc. and permission has been granted for this version to appear in e-Publications@Marquette. Lippincott Williams \& Wilkins, Inc. does not grant permission for this article to be further copied/distributed or hosted elsewhere without the express permission from Lippincott Williams \& Wilkins, Inc.

\section{Histologic Evaluation of the Efficacy of rhBMP-2 Compared With Autograft Bone in Sheep Spinal Anterior Interbody Fusion}

\section{Sandhu, Harvinder S.}

Department of Orthopaedic Surgery and UCLA Comprehensive Spine Center, UCLA School of Medicine, Los Angeles, CA

Spine Service Hospital for Special Surgery, Cornell University Medical College, New York, NY Toth, Jeffrey M.

Section of Biomaterials Research, Department of Orthopaedic Surgery, Medical College of Wisconsin, Milwaukee, WI

Diwan, Ashish D.

Spine Service Hospital for Special Surgery, Cornell University Medical College, New York, NY

St. George Hospital, University of New South Wales, Sydney, New South Wales, Australia

Seim, H. B. III

Department of Clinical Sciences, College of Veterinary Medicine and Biomedical Sciences, Colorado State University, Fort Collins, CO

Kanim, Linda E. A. 
Department of Orthopaedic Surgery and UCLA Comprehensive Spine Center, UCLA School of Medicine, Los Angeles, $\mathrm{Ca}$

Kabo, J. Michael

Department of Orthopaedic Surgery and UCLA Comprehensive Spine Center, UCLA School of Medicine, Los Angeles, $\mathrm{Ca}$

Turner, A. Simon

Department of Clinical Sciences, College of Veterinary Medicine and Biomedical Sciences, Colorado State University, Fort Collins, CO

\section{Keywords:}

spinal fusion; anterior lumbar; cage; titanium; rhBMP-2; sheep; histology

\section{Abstract:}

Study Design. The sheep anterior lumbar spinal fusion model was used to study the efficacy of recombinant human bone morphogenetic protein-2 (rhBMP-2)-collagen composite in comparison with autograft to enhance spinal interbody fusion. Comparisons were drawn from temporal radiographic and end-point biomechanical and histologic data.

Objective. To analyze histologically the ability of rhBMP-2 to achieve complete arthrodesis between vertebral bodies.

Summary of Background Data. Studies using rhBMP for enhancement of anterior interbody fusion have used numerous endpoints. However, systematic histologic evaluation of the fusion has not been conducted.

Methods. Twelve sheep underwent single-level anterior lumbar interbody fusion performed with a cylindrical fenestrated titanium interbody fusion device (INTER FIX, Medtronic Sofamor Danek, Inc., Memphis, TN). The device was filled either with rhBMP-2-collagen $(n=6)$ or autogenous iliac crest bone graft $(n=6)$. Radiologic evaluation was carried out at 2-month intervals, and all sheep were killed 6 months after surgery. Nondestructive biomechanical testing for stiffness to flexion, extension, and lateral bending moments, un-decalcified histology, and qualitative and quantitative histologic evaluation were performed.

Results. Radiographs revealed a bony bridge anterior to the cage in five of six rhBMP-2-treated animals, whereas it was present only in one of five in the autogenous bone graft group. Segments treated with rhBMP-2 were $20 \%$ stiffer in flexion than autograft-treated segments at 6 months. Six of six in the rhBMP-2 group and two of six in the autograft group showed complete fusion. There was a significantly higher rate of bony continuity observed at the fenestrations of the rhBMP-2 group. Three times more number of cage fenestrations in the rhBMP-2 group demonstrated "all-bone" when compared with the autograft group $(P<0.001)$. Further, the scar tissue in and around the autografttreated cages was 16 -fold more $(P<0.01)$ than that seen for rhBMP-2-treated cages. 
Conclusions. The study demonstrates that rhBMP-2 can lead to earlier radiologic fusion and a more consistent increased stiffness of the segments when compared with autograft in sheep anterior lumbar interbody fusion. Furthermore, a three times higher histologic fusion rate is attainable with significantly reduced fibrous tissue around the implant when rhBMP-2 is used.

Among the growth factors that have shown most promise in bone formation and regeneration are the low molecular weight bone morphogenetic proteins (BMPs). More than 24 different types of BMPs have been identified; however, BMP-2, -4 , and -7 (also known as osteogenic protein-1) are the most studied osteoinductive factors. BMPs have found clinical application in spinal surgery specifically in anterior spinal fusion. Marshall Urist 8 reported their discovery in 1965, and by the end of the century one of these BMPs (recombinant human BMP-2, rhBMP-2) has been shown to be of clinical benefit for patients with degenerative disc disease when used for anterior lumbar interbody fusion (ALIF). $\underline{2}$

ALIF is one of the surgical options available for managing refractory degenerative disc diseases. Of the many devices available for ALIF, cylindrical and fenestrated cages have been developed with an intent of providing immediate anterior intervertebral fixation. These devices simultaneously facilitate osseous bridging across the intervertebral space. 1 The design of these devices is predicated on the distractioncompression concept originated by Bagby, whereby the diseased disc space is first prepared and distracted to place the anulus under maximum tension. $\underline{5}$ The threaded, hollow, and cylindrical device is then screwed into a reamed hole, permitting thread purchase of subchondral bone of both adjacent vertebrae. The result is a circumferential tension band imposed by an intact but distracted anulus on a rigid, internally fixed device. However, the clinical use of these devices at this time has not precluded the requirement for autologous iliac crest bone harvest and grafting to facilitate osseous fusion.

Autogenous bone graft obtained from the iliac crest has major disadvantages in several clinical situations. These include significant postoperative morbidity at the donor site in $8 \%$ of patients (e.g., infection, pain, hemorrhage, nerve injury), increased operative time, and additional cost. 9 Strategies devised to obviate the need for autologous bone harvest during ALIFs include the following: 1) development of uniformly porous metallic cylindrical fusion devices, simulating the architecture of cancellous bone to facilitate bone ingrowth and anchorage, and 2) use of potent stimulators of bone formation with growth factors such as rhBMP-2 or rhBMP-7.

We have deployed both strategies over the years. Specifically, we have shown that the interbody height index is better maintained by a titanium-based, threaded, hollow, and fenestrated interbody fusion cage containing autogenous bone graft when compared with autologous Cloward-type bone dowel grafts alone in an in vivo sheep lumbar spinal fusion model. $\underline{7}$ We have also used rhBMP-2collagen sponge composite in a rhesus monkey laparoscopic ALIF model and showed that at 24 weeks after surgery monkeys receiving rhBMP-2 developed solid intervertebral fusion, whereas monkeys receiving the carrier alone did not fuse as assessed by manual palpation, CT scan, or histology. $\stackrel{1}{=}$ We have recently reported data on an ALIF human clinical trial in a preliminary manner wherein we showed that patients treated with rhBMP-2-filled titanium cages for a single-level fusion achieved radiologic arthrodesis in a more reliable manner when compared with patients receiving autograft-

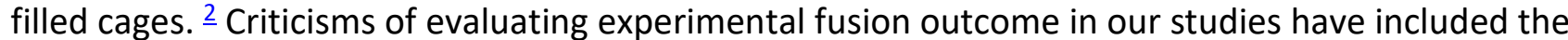
following: 1) radiologic evaluation of fusion across intervertebral segment through the fusion device is 
difficult and 2) stiffness of intervertebral body segments as measured biomechanically or by manual palpation can also be an index of fibrous scarring. Therefore, histology of the fusion may be the best index of progression or completion of intervertebral fusion.

Intervertebral fusion histology has not been systematically studied in an effort to evaluate the efficacy of rhBMP-2-collagen sponge carrier alone when compared with autograft bone in achieving spinal fusion. Here, our hypothesis was that a significantly better histologic intervertebral bony fusion can occur with the use of rhBMP-2 to fill a titanium cylinder when compared with autologous bone graft. In this study we have examined temporal radiologic, endpoint mechanical, and histologic properties of a threaded, titanium fusion cylinder filled with either autologous bone graft or with rhBMP-2 in a sheep ALIF model.

\section{Materials and Methods}

\section{Materials}

Threaded Titanium Fusion Device.

The interbody fusion cage (INTER FIX, Medtronic Sofamor Danek, Inc., Memphis, TN) was made of a titanium alloy (Ti-6Al-4V) and fabricated as a closed hollow cylinder. The device had an external diameter of $14 \mathrm{~mm}$ and contained a screw-in end cap to allow for placement of graft materials. The device porosity as described by the manufacturer was $35 \%$ overall hole-to-metal ratio with the porosity distributed circumferentially for contact with the intervertebral bodies. The quality inspection data from the manufacturer indicated that the mechanical load to yield was $80,000 \mathrm{~N}$ (maximum human physiologic loads $\sim 10,000 \mathrm{~N}$ ), and cyclic compressive loading from 800 to $9680 \mathrm{~N}$ at $15 \mathrm{~Hz}$ over $5,000,000$ cycles resulted in no observable microscopic damage or deformation.

rhBMP-2.

The rhBMP-2 was produced by a Chinese hamster ovarian cellular expression system by Genetics Institute (GI Inc., Andover, MA) and was supplied by Medtronic Sofamor Danek, Inc. (Memphis, TN). rhBMP-2 was provided in a freeze-dried form and reconstituted at the time of surgery with a buffer solution to a final concentration of $0.43 \mathrm{mg} / \mathrm{mL}$. One milliliter of rhBMP-2 solution was drip applied onto a commercially available $2.5 \mathrm{~cm} \times 5 \mathrm{~cm}$ Type I collagen sponge (Absorbable Collagen Hemostatic Sponge, Helistat) (Integrated Life Sciences, Plainsboro, NJ) and allowed to bind to the sponge for 30 minutes, after which this composite was inserted into the titanium cages.

\section{Animal Model and Experimental Design.}

Mature female merino sheep with an average ( \pm SD) weight of $65( \pm 2.4)$ kg underwent a single-level midlumbar anterior interbody fusion from April 1995 to July 1995 using our operating procedure as previously described. ${ }^{7}$ A left anterior retroperitoneal approach was used, and the intervertebral disc between $L 4$ and $L 5$ was exposed. A core of the L4-L5 disc along with $3 \mathrm{~mm}$ of the adjacent endplates was prepared from the left lateral side in such a manner that the untapped core could accept the 14$\mathrm{mm}$ threaded, self-tapping titanium cylinder. At this point experimental group allocation was done. 
The sheep received either a titanium cage with rhBMP-2-impregnated collagen $(n=6)$ or a titanium cage with morcellized corticocancellous autograft harvested from the left iliac crest through the same incision $(n=6)$. The surgical wound was closed in a standard manner, $\underline{7}$ and the sheep were allowed to graze immediately after surgery without any external immobilization. All sheep were killed 6 months after surgery. Spinal columns from L3 to L6 were dissected with retention of intersegmental ligamentous tissues, and specimens were stored at -20 degrees $C$ until further evaluation. Weightmatched unoperated cadaver sheep spines were studied to determine the mechanical stiffness of untreated spinal segments $(n=14)$. The animal protocol was approved by the institutional ethics committee of Colorado State University.

\section{Radiographic Evaluation of Fusion Status.}

Under general anesthesia, lateral radiographs of the lumbar spine were obtained immediately after surgery, 2 and 4 months after surgery, and after death. Bone outside (and anterior to) the cage and whether or not it was bridging were evaluated (Figure 1). A score was assigned depending on absence (Grade 0 ) or presence of bone anterior to the cage. When bone was present and there was an attempt at bridging from one side only, that sample scored Grade 1. When there was "attempted" bridging from both sides, the sample was assigned Grade 2, and Grade 3 was assigned if the attempted fusions from the cephalad and caudad ends of the vertebrae were one continuous mass but this mass did not project beyond the anterior margin of the vertebral bodies. When the fusion mass extended in front of the anterior margins of the contiguous vertebrae, this was assigned a Grade 4 (Figure 1). The observer (A.D.D.) was blinded to the groups when scoring the radiographs. Radiographs were available for five animals in the autograft group.

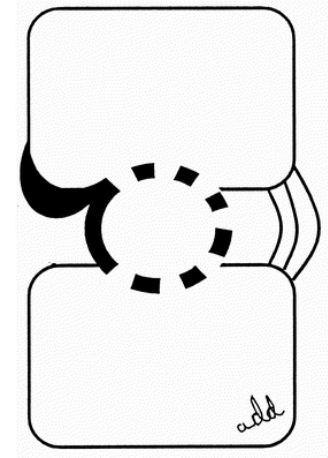

1

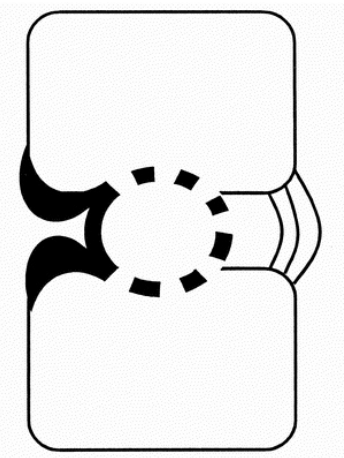

2

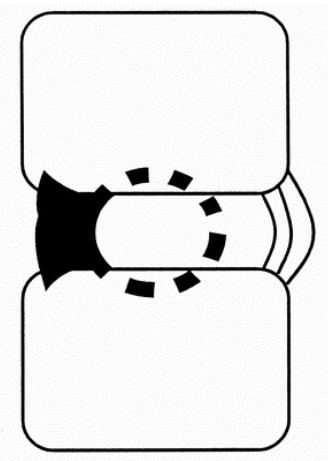

3

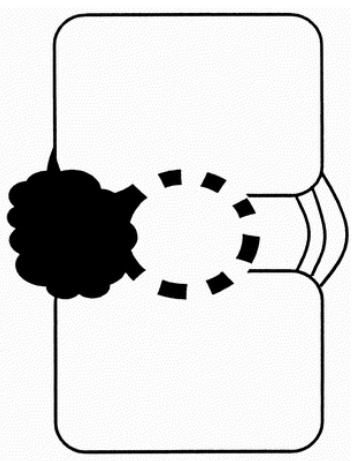

4

Figure 1

Mechanical Evaluation of Fusion.

The L4-L5 intervertebral segments (fusion sites) were tested for stiffness to sagittal and coronal plane bending moments (flexion, extension, right bending, left bending) for the two operated groups and for 
14 unoperated cadaver sheep spines. Transverse processes of the dissected specimens were trimmed to facilitate polymethylmethacrylate potting of the L3 and L6 vertebrae. The polymethylmethacrylate pots did not include the L3-L4 or the L5-L6 discs. Nondestructive mechanical tests were performed with an MTS 812 servohydraulic testing machine. The specimen was mounted in the apparatus in the horizontal plane, so that it was oriented perpendicular to the axis of actuation. The proximal end of the specimen was fixed, whereas the distal end was free to move and was placed directly above the actuator. Pure bending moments were applied using a system of cables and pulleys (Figure 2). Rotational variable differential transformers were attached to the vertebral body via bone screws to measure rotation in the L4-L5 motion segment and to the free end to measure its angle with respect to horizontal as described previously. $\underline{6}$ Load-displacement data were recorded. For each test, loads were applied in three cycles consisting of a 5-second ramp per cycle with a maximum applied moment of $10 \mathrm{Nm}$. Tests were performed in flexion, extension, right bending, and left bending modes sequentially. All loadings were nondestructive, resulting in a linear load versus deformation response. Stiffness was calculated as the slope of the force versus deformation response and converted to the appropriate units according to the type of test conducted. All tests were conducted within the elastic range.

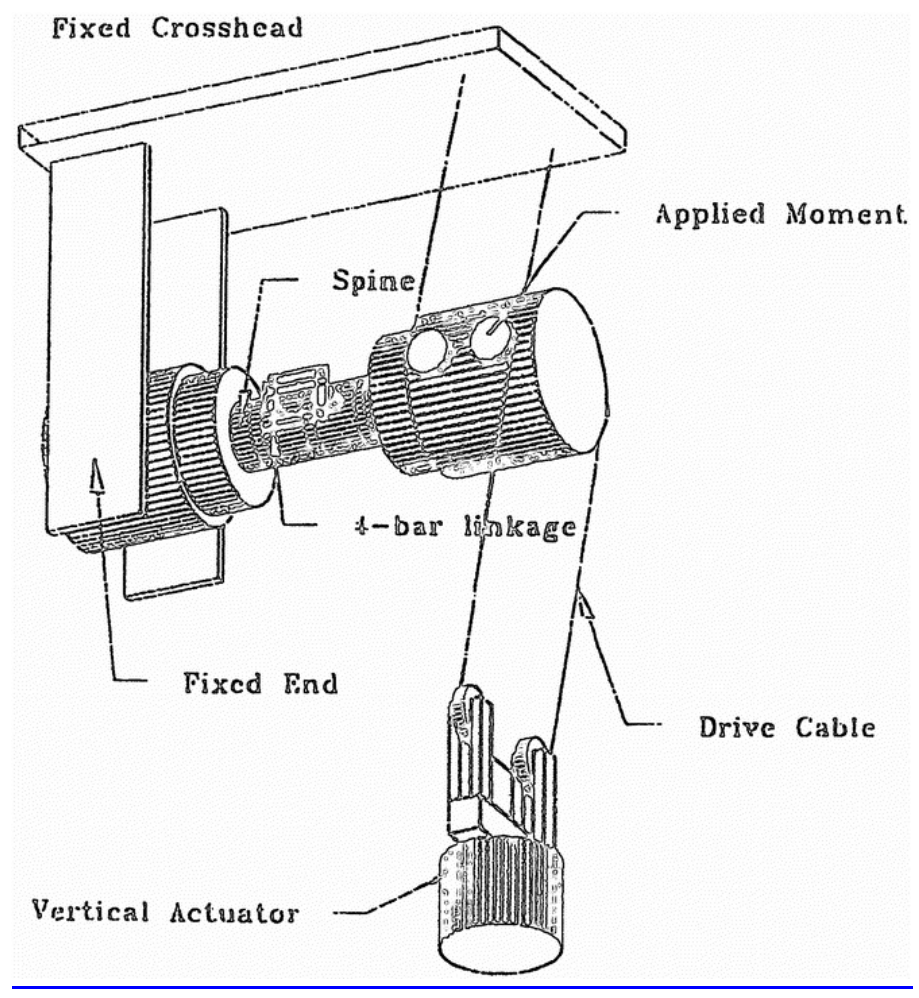

Figure 2

\section{Fusion Histology.}

After mechanical testing the spinal motion segment were fixed in $70 \%$ ethyl alcohol and bisected in the sagittal plane for histologic analysis. Both halves of the spinal segment were subjected to un- 
decalcified histologic analyses, consisting of toluidine blue-O and basic fuchsin staining. After sufficient time had passed to effect fixation, the implants and tissues were sequentially dehydrated in $95 \%$ alcohol for 4 days, 2 changes of 100\% alcohol for 2-3 days each, and finally cleared in xylene for 1 day. Next, these explants were sequentially infiltrated in graded catalyzed methyl methacrylate. After polymerization the blocks were trimmed and sectioned on a diamond saw (Isomet Low Speed Saw, Buehler, Lake Bluff, IL) to produce at least 10 sections of 100- to 200- $\mu$ m thickness from both halves. Both the right and left halves were sectioned in the sagittal plane from medial to lateral until the end of the device was reached.

Differential staining with basic fuchsin and toluidine blue-O was performed on all of the un-decalcified sections after microradiographs were produced to determine the quality and quantity of osteogenesis in contact with the device and in the open fenestrations of the titanium device as well as to evaluate the histologic host response to the titanium device with the autograft or rhBMP-2-collagen sponge composite.

\section{Histologic Analysis.}

After staining, all un-decalcified sagittal sections and microradiographs were read by an orthopedic histopathologist (J.M.T.) in a blinded fashion. The nature of the fusion was commented on after a detailed analysis of 10 randomly selected un-decalcified stained sagittal sections of the fusion level for separate animals. Evaluation included qualitative assessment of osteogenesis in contact with the device and in the open pores of the titanium device as well as the histologic and cytologic host response in the vicinity of the titanium device. In each sagittal section the presence of intervertebral fusion anterior to, posterior to, or through the device was determined as follows: 1) an uninterrupted bridge of bone present in the anterior margin was considered an anterior fusion, 2) an uninterrupted bridge of bone present in the posterior margin was considered posterior fusion, or 3) continuous bone ingrowth from the endplate of the cephalad vertebrae through the superior, middle, and inferior portions of the device and into the endplate of the caudal vertebrae was considered fusion through the device. Based on these criteria, each specimen was assigned an overall rating of fusion as follows: 1 ) complete fusion, 2) partial or incomplete fusion, or 3) nonfusion. A rating of complete fusion was given if the majority of sections of a specimen depicted a complete intervertebral bridge of bone. If intervertebral bridging was present either through the metallic device or anterior or posterior to it but was noted in less than a majority of sections, then a rating of partial fusion was given. If no sections depicted a complete, uninterrupted, intervertebral bridge of bone, then the sample was rated as nonfusion.

After the analysis by the orthopedic pathologist, sections for each sample that were representative of the fusion status were selected, photographed, and further analyzed in a blinded fashion by one of the investigators (A.D.D.). To study the continuity of bone inside the cage (intracompartmental) with that outside the cage (extracompartmental), bony continuity was evaluated at the fenestrations of the cage in sagittal sections ( $\underline{\text { Figure } 3}$ ). Specifically, a trapezoidal template was drawn centering on each fenestration of the titanium cage and to include the contiguous intracompartment and extracompartment regions measuring half the thickness of the cage from the respective inner or outer rim of the cage (Figure 3). A fenestration was labeled "all bone" if only bone was observed (blue 
stained tissue), "partial bone" if there was presence of a mixture of bone and fibrous tissue (pink stained tissue), or "no bone" if only fibrous tissue was seen. Results were expressed as a percentage for each sample.

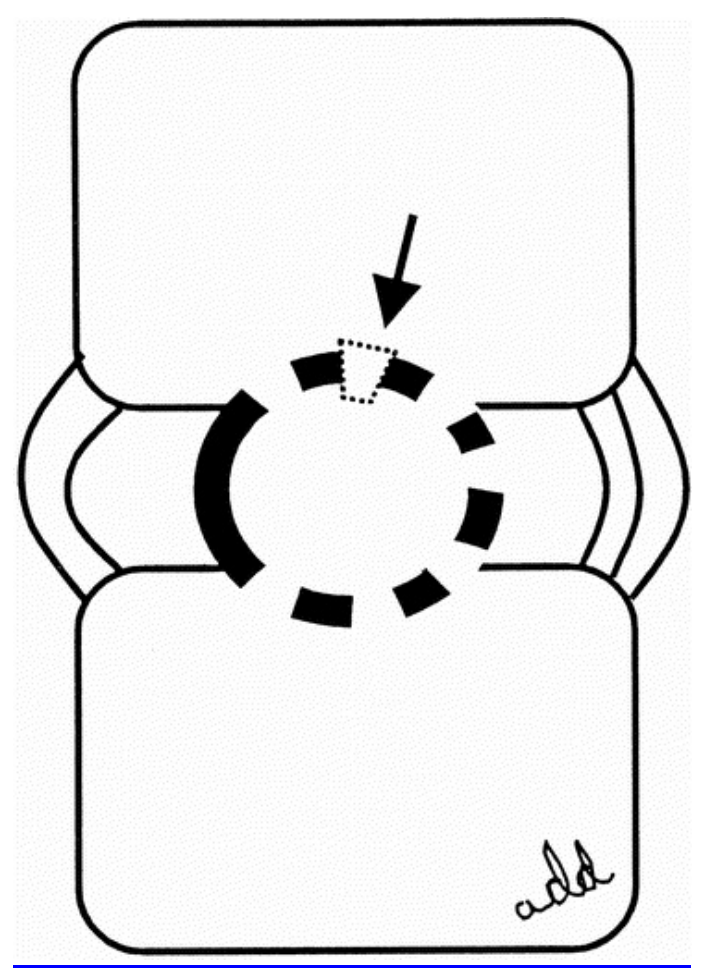

\section{$\underline{\text { Figure } 3}$}

It was thought that the quantity of new bone in the two groups was not comparable because the autograft group sections showed presence of new bone, revascularized graft, and necrotic autograft. One observer (A.D.D.) evaluated the amount of fibrous tissue in and around the cage in a blinded fashion as an index of poor fusion (more fibrous tissue = poorer histologic fusion). The MetaMorph version 4.0 image analysis system (Universal Imaging Corp., West Chester, PA) was used. A rectangular grid was overlaid around the cage that was tangential and touching the caudad and cephalad outer margins of the cage and extending two cage thicknesses outside the anterior and posterior margins (Figure 4). Percentage areas representing the cage, fibrous tissue (pink), and bony tissue (blue-purple) were estimated for each representative section in both groups of animals. 


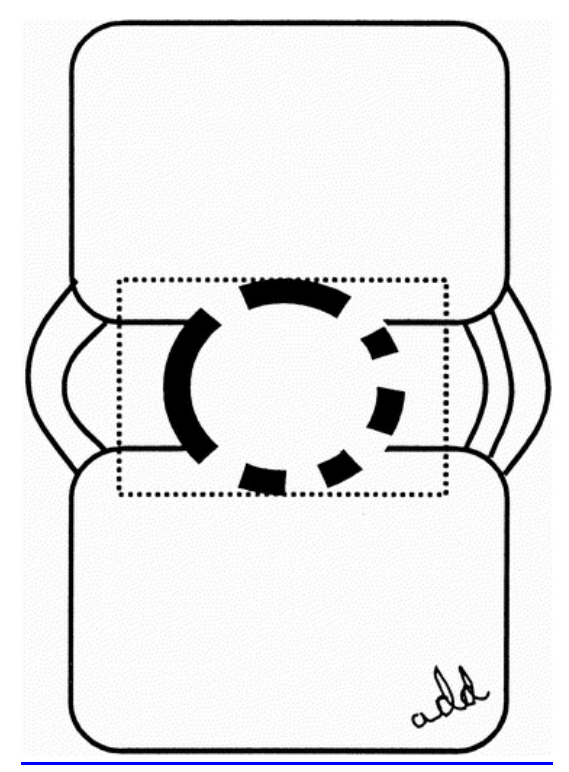

Figure 4

\section{Statistical Analysis.}

All observations are reported as averages ( \pm SEM). Repeated-measures analysis of variance on ranks was performed for sentinel scores with pairwise multiple comparison within groups (Dunnett's method). Analysis of variance was also applied to mechanically tested stiffness measures for each plane of motion for each group. Fischer exact test was applied to compare overall histologic fusion. An unpaired Student's $t$ test was applied to compare bone in fenestrations and fibrous tissue area between the autograft and rhBMP-2-treated groups. A statistically significant observation was reported when $P \leq 0.05$.

\section{Results}

Radiographs revealed a bony bridge anterior to the cage in five of six rhBMP-2-treated animals, whereas it was present in only one of five in the autogenous bone graft group. Radiologic evidence of union was absent in both groups immediately after the operation (week 1). At 6 months after the ALIF with titanium cage the average $( \pm$ SEM) radiologic score was $1.4(0.5)$ for the autograft group, whereas it was nearly double for the rhBMP-2 group at 2.6 (0.4) (Figure 5). When compared with immediately after surgery, the autograft group showed definite fusion only at 6 months $(P<0.05)$, whereas the rhBMP-2-treated interbody showed fusion as early as 8 weeks $(P<0.01)$, which was well sustained at 16 weeks $(P<0.01)$ and at 6 months $(P<0.05)$ (Figure 5$)$. 


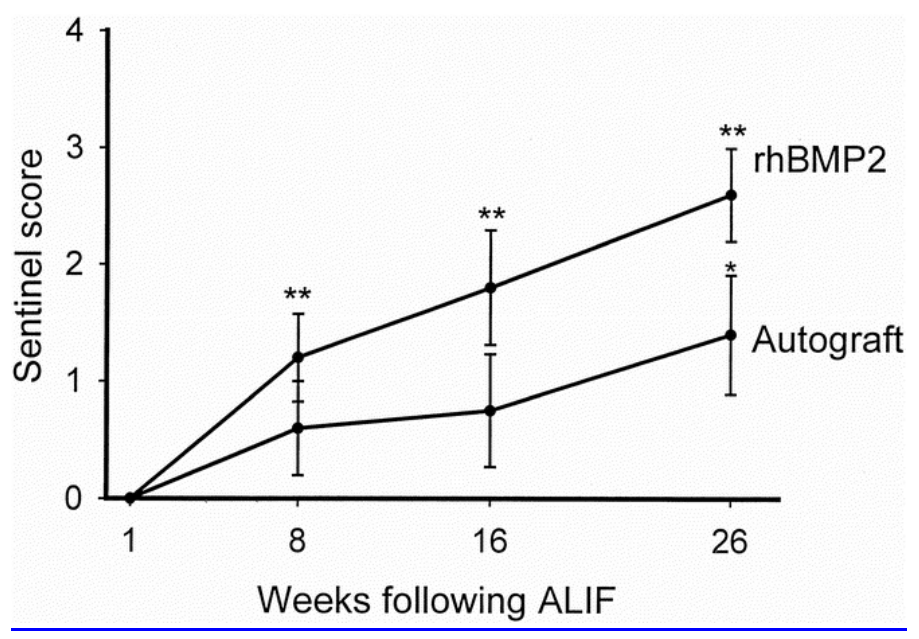

Figure 5

All specimens that had undergone the ALIF procedure in both groups were stiffer than unoperated controls. The autograft group was five times stiffer than the unoperated controls in left bending $(P<$ 0.05). No significant differences from unoperated control were found with other loading modes for this group. The rhBMP-2 group was significantly stiffer in bending in all directions compared with the unoperated controls. This group demonstrated a 2.5 -fold increase in stiffness in flexion $(P<0.001)$, a fourfold increase in extension $(P<0.001)$, a sixfold increase in right bending $(P<0.001)$, and a fourfold increase in left bending (Figure 6 ) relative to the control group. On comparing stiffness between the autograft and rhBMP-2 groups, no statistical difference was found for mechanical stiffness between the autograft and rhBMP-2 groups for any of the loading modes.

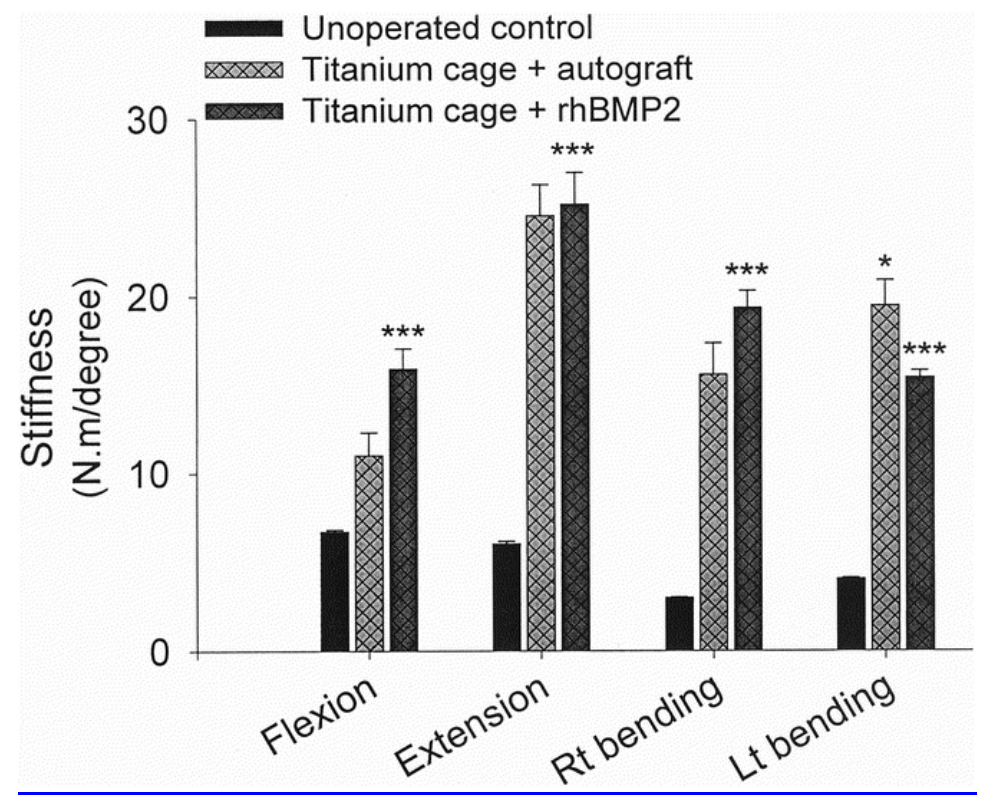

\section{Figure 6}


Our staining technique stained bone blue-green with the osteoid seams as blue and the unmineralized osteoid as red. Cartilage tissue and fibrocartilage stained dark purple, and fibrous tissue stained pink or pink-red. A typical un-decalcified stained sagittal section from the autograft group showed the following features: Sections mostly failed to demonstrate anterior or posterior bridging bone. The adjacent outer and inner surfaces of the titanium cage would show fibrous or fibrocartilaginous tissue. There was good bone contact caudad and cephalad. However, the interior of the cage contained some new bone, bony fragments, fibrocartilage, and fibrous tissue. There was evidence of resorption of the graft material; however, many sections failed to demonstrate resorption cavities in the central regions of the cage, indicating a decrease in osteoclastic activity (Figure 7).
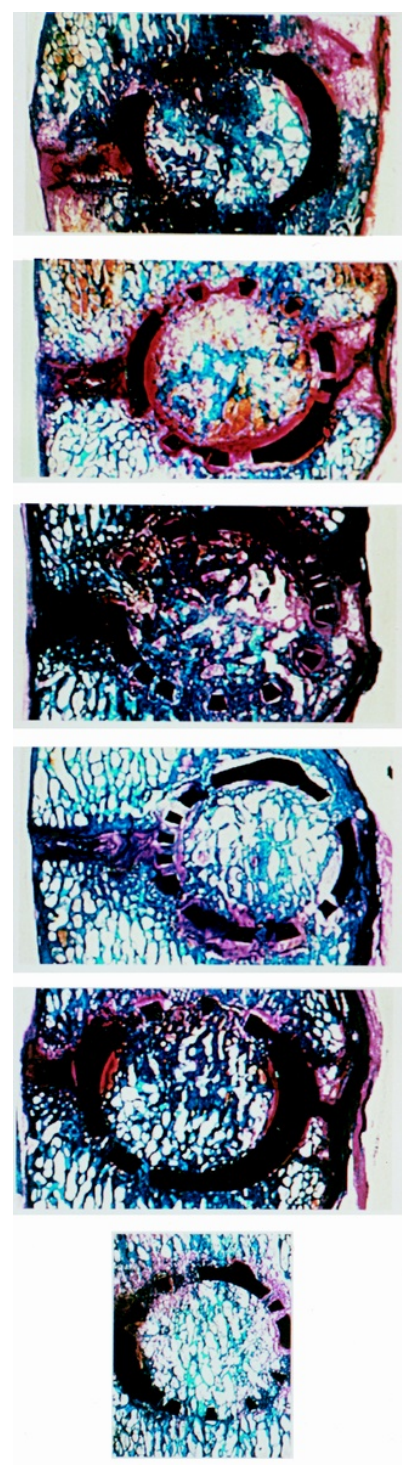

A

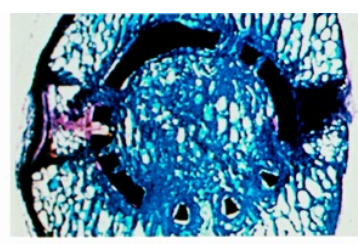

B

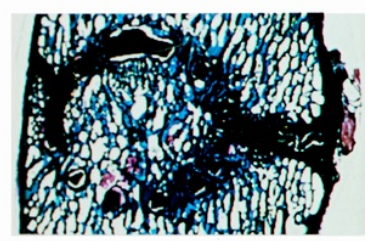

C

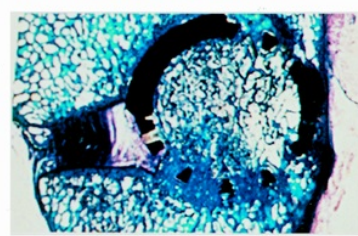

D

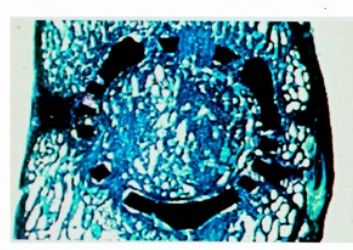

E

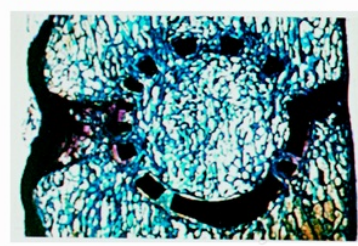

F

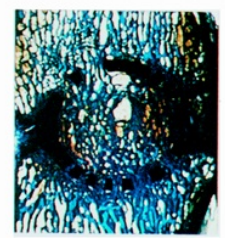

Figure 7 
Sections belonging to animals that had an rhBMP-2-collagen composite placed in the cage 6 months earlier were typified by bony bridging found in the region of the device. Some of the sections demonstrated trabecular bridging outside the device without any evidence of pseudarthrosis. Inside the cage there was no evidence of presence of the collagen carrier. Second, extensive trabecular bone was found to grow through the cage in all sections. Third, no evidence of enchondral or intramembranous ossification was seen at the 6-month time point, indicating a completion of the process of bone formation. The new bone formed was a mixture of primary and secondary osteons with little woven bone observed. Trabeculae were organized and consolidated into coarse cancellous bone structure. The bone within the cage appeared viable as osteocytic lacunas were occupied by osteocytes.

There were four animals in the autograft group that did not achieve fusion at the operated site according to the overall fusion criteria. The rhBMP-2 group all displayed complete fusion. There was a fivefold increase in the rate of fusion in the rhBMP-2 group (100\%) when compared with the control autograft group (20\%) 6 months after the ALIF procedure.

There was a significantly higher rate of bony continuity observed at the fenestrations of the rhBMP-2 group. Three times as many of the cage fenestrations in the rhBMP-2 group demonstrated all bone when compared with the autograft group $(P<0.001)$. Partial bone filling $(P<0.001)$ or absence of bone was observed on only $20 \%$ of the occasions for the rhBMP-2 group compared with the autograft group (Figure 8).

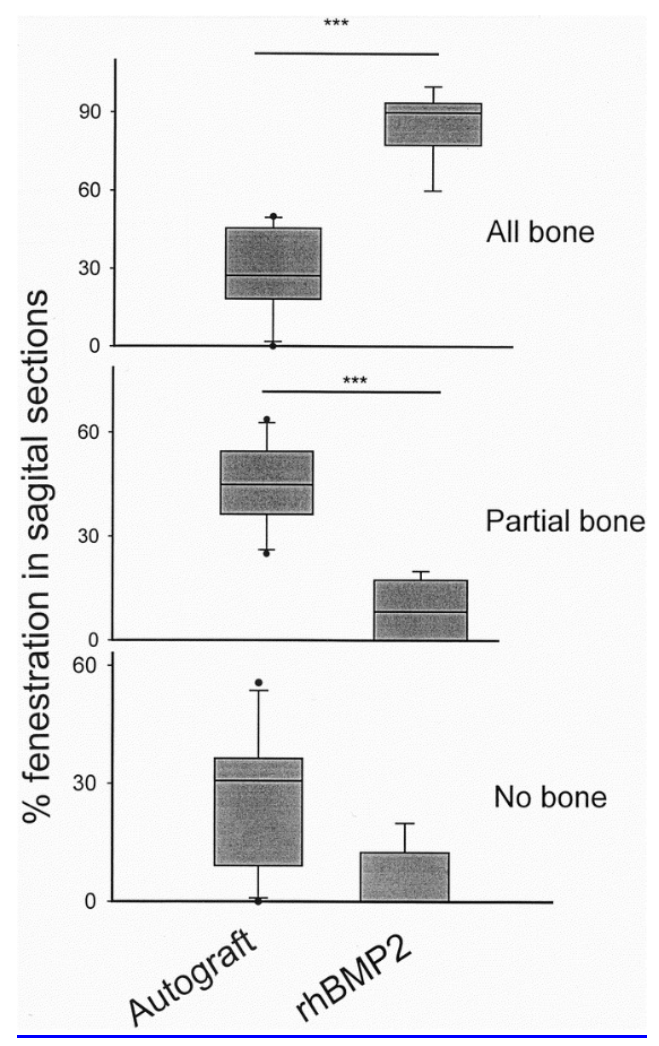


Figure 8

The autograft group demonstrated a 16-fold greater area of fibrous tissue in the sagittal sections compared with the rhBMP-2 group $(P<0.01)$.

\section{Discussion}

We show conclusively that histologic fusion after a titanium cage stabilized ALIF in sheep is significantly superior if a single dose of rhBMP-2 is used within the cage during surgery when compared with the use of a cage filled only with autograft. A 16-fold greater fibrous tissue formation in the autograft group at 6 months after ALIF demonstrated the potency of rhBMP-2 in inducing cellular components within the region of fusion to differentiate into bone.

A clinically significant overall fusion rate, as determined by reviewing multiple sagittal sections of the same sample, was 5 times greater for the rhBMP-2 group compared with the autograft group (Table 1). The issue of evaluating bony continuity from the cephalad to caudad vertebra through the cage device was overcome by the use of a novel method of estimating the bridging bone at the fenestrations (Figure 3). There was a significantly threefold higher incidence of bony bridging at the fenestrations in the rhBMP-2 group (Figure 8). The absence of any evidence of the process of new bone formation including either enchondral ossification or membranous ossification in the rhBMP-2 group indicated that the process had been completed before the deaths of the animals at 26 weeks. However, in the autograft group the process of creeping substitution of the autografts was evident, and in some cases this process had ceased in the central regions of the cage.

\begin{tabular}{l} 
Table 1. The Overall Histologic Fusion Rate at 6 Months \\
for ALIF Performed in Sheep Using a Titanium Cage \\
Either With Autograft or Collagen Impregnated With \\
rhBMP-2 \\
\hline Fusion Rating \\
\hline Complete \\
Incomplete \\
Nonfusion \\
\hline Each specimen was given an overall rating of fusion as: 1) complete fusion, 2) \\
partial or incomplete fusion, or 3 ) nonfusion. A rating of complete fusion was \\
given if the majority of sections of a specimen depicted a complete interver- \\
tebral bridge of bone. If intervertebral bridging was present either through the \\
titanium device or anterior or posterior to it but was noted in less than a \\
majority of sections, then a rating of partial fusion was given. If no sections \\
depicted a complete, uninterrupted, intervertebral bridge of bone, then the \\
sample was rated as nonfusion. For the benefit of statistical analysis interver- \\
tebral body fusion was rated as either fused or not fused and the Fisher Exact \\
test applied to compare the two groups. This revealed that there was no \\
significant difference in the proportions of samples fused in the rhBMP-2 \\
group ( $P<0.06$ ).
\end{tabular}




\section{Table 1}

On careful review of prior studies conducted to enhance ALIFs using rhBMP, it was found that histology of the fusion mass was not systematically evaluated either quantitatively or qualitatively (Table 2). Our histologic results demonstrate a definite enhancement of spinal fusion by rhBMP-2 over the use of autograft. Zdeblick et al $\underline{10}$ performed an elegantly designed study to evaluate the efficacy of rhBMP-2 compared with autograft in achieving a BAK cage-stabilized cervical interbody fusion in goats. Their microradiographic scoring system awards a high score to bone within the cage. As autograft and de novo new bone, both would have cast radiodense shadows, and the autograft group would have inadvertently scored high. Despite this potential problem, Zdeblick et al demonstrated a significantly higher score for the rhBMP-2 group. 10 In the histomorphometric analysis of fusion in the work by Cunningham et al, $\underline{3}$ the bone within the cage was estimated using image analysis. This again leads to the inability to judge the contributions of autograft (old bone) and de novo (new bone) to this histomorphometric estimation. In the present study we did not quantify bone within the cage because necrotic autograft also stained a similar color as new bone. Our quantification of pink-stained fibrous tissue as an index of poor progression of fusion overcomes the aforementioned limitation of using microradiographs for estimation of de novo new bone formation (Figure 4).

Table 2. Outcome Measures Used in rhBMP Aided Anterior Interbody Fusion Studies

\begin{tabular}{|c|c|c|c|c|c|}
\hline Investigators & Species & Level & Groups & Device & Measured \\
\hline Boden et $\mathrm{al}^{2}$ & Human & Lumbar & BMP-2/autograft & Novus LT & $\begin{array}{l}\text { Duration of surgery } \\
\text { X-rays } \\
\text { Clinical }\end{array}$ \\
\hline Boden et $\mathrm{al}^{1}$ & Rhesus monkey & Lumbar & BMP-2/carrier alone & $\begin{array}{l}\text { Threaded hollow Ti cylinder } \\
\text { (custom) allograft }\end{array}$ & CT scan \\
\hline Hecht et al ${ }^{4}$ & Rhesus monkey & Lumbar & BMP-2/autograft & Freeze dried bone dowels & $\begin{array}{l}\text { X-ray } \\
\text { Manual palpation } \\
\text { Descriptive histology }\end{array}$ \\
\hline Zdeblick et $\mathrm{al}^{10}$ & Alpine goats & Cervical & BMP-2/autograft & BAK-Ti cylinder & $\begin{array}{l}\text { X-ray } \\
\text { Microradiograph } \\
\text { Fluorochrome } \\
\text { Biomechanics }\end{array}$ \\
\hline Cunningham et $\mathrm{al}^{3}$ & Sheep & Thoracic & RhOP1/autograft & BAK-Ti cylinder & $\begin{array}{l}\text { Biomechanics } \\
\text { CT scan } \\
\text { Histomorphometry }\end{array}$ \\
\hline
\end{tabular}

Table 2

We have also demonstrated that radiologic progression of fusion is achieved at 8 weeks in the rhBMP2 -treated cage group versus 26 weeks for the autograft-treated cage group. This shows that rhBMP-2 enhances the rate at which spinal fusion can be achieved in a sheep ALIF model. The use of the radiologic scoring method to assess fusion in this study is restricted in its ability to estimate all bridging bone between the two vertebrae because Grades 1 and 2 are essentially nonfusions, whereas Grades 3 and 4 are fusions. In the present study we achieved good discrimination temporally for the radiographic score within both groups (igure 5 ). 
Mechanical stiffness for the rhBMP-2-treated group was more consistent in all directions, whereas in the autograft group there was a significant difference in stiffness when compared with the unoperated control segments only on left bending (Figure 6). All cages were inserted from the left side in both groups; hence, one may expect some decrease in stiffness on right bending if the fusion on the left side is not complete.

Furthermore, the laterally oriented cages in this study may simulate a more challenging environment because of the potential motion in the flexion-extension plane compared with a more clinically comparable positioning of the cages in the anteroposterior orientation. It is possible that this mechanical disadvantage is reflected as a poor rate of bony healing and combined with substantial fibrosis in the autograft group.

We have not presented any data related to the duration of surgery or to early and late functional outcomes in the sheep. Hence, it is not possible to comment on the superiority of avoiding autograft procurement procedure for our rhBMP-2-treated group. Furthermore, it may be opportune to point out here that in a clinical setting anterior spinal fusion using iliac bone graft will have an $80-90 \%$ fusion rate. The low fusion rate in our study may reflect the stringent histologic criteria applied to evaluate the arthrodesis in our sheep model versus use of radiologic data and final clinical outcomes used to evaluate fusion in a clinical setting.

Our present work compliments our previous work and the work of others by demonstrating definite and direct evidence of interbody fusion enhancement by rhBMP-2. Because rhBMP-2 is already in clinical use, our present results increase the confidence in the reliability and efficacy of rhBMP-2 to achieve solid spinal interbody fusion. This work has also demonstrated that rhBMP-2 can accelerate the rate of spinal fusion, and it remains to be answered by future investigations whether this biologic potentiation of fusion by rhBMP-2 will make posterior mechanical stabilization of ALIFs redundant.

\section{Key Points}

- Previous studies dealing with spinal interbody fusion enhanced by the use of BMPs have used several outcome measures.

- True bony fusion or bony arthrodesis across two spinal segments can best be evaluated by histology.

- This study assesses bone bridging across spinal segments using systematic histologic evaluation.

\section{Acknowledgment}

The authors thank Stephen Doty, PhD, from the ultrastructural microscopic section of Bone and Mineral Research at the Hospital for Special Surgery for facilitating image analysis.

\section{References}

1. Boden SD, Martin GJ, Horton WC, et al. Laparoscopic anterior spinal arthrodesis with rhBMP-2 in titanium interbody threaded cage. J Spinal Disord 1998; 11: 95-101. 
2. Boden SD, Zdeblick TA, Sandhu HS, et al. The use of rhBMP-2 in interbody fusion cages: definitive evidence of osteoinduction in humans-a preliminary report. Spine 2000; 25: 376-81.

3. Cunningham BW, Kanayama M, Parker LM, et al. Osteogenic protein versus autologous interbody arthrodesis in the sheep thoracic spine: a comparative endoscopic study using the Bagby and Kuslich interbody fusion device. Spine 1999; 24: 509-18.

4. Hecht BP, Fischgrund JS, Herkowitz HN, et al. The use of recombinant human bone morphogenetic protein-2 (rhBMP-2) to promote spinal fusion in a nonhuman primate anterior interbody fusion model. Spine 1999; 24: 629-36.

5. Kuslich SD, Ulstrom CL, Griffith SL, et al. The Bagby and Kuslich method of lumbar interbody fusion: history, techniques, and 2-year follow-up results of a United States prospective, multicenter trial [see comments]. Spine 1998; 23: 1267-78; discussion 1279.

6. Markolf K, Delamarter R, Fyodorov I, et al. Variables affecting pedicle screw plate fixation of an unstable L3-L4 defect. Clin Orthop 1996; 327: 283-90.

7. Sandhu HS, Turner S, Kabo JM, et al. Distractive properties of a threaded interbody fusion device: an in vivo model. Spine 1996; 21: 1201-10.

8. Urist MR. Bone: formation by autoinduction. Science 1965; 150: 893-9.

9. Younger EM, Chapman MW. Morbidity at bone graft donor sites. J Orthop Trauma 1989; 3: 192-5.

10. Zbeblick TA, Ghanayem AJ, Rapoff AJ, et al. Cervical interbody fusion cages: an animal model with and without bone morphogenetic protein. Spine 1998; 23: 758-66. 